\title{
Phase diagram of a ternary mixture of cholesterol and saturated and unsaturated lipids calculated from a microscopic model
}

\author{
R. Elliott $^{1}$, I. Szleifer ${ }^{2}$, M. Schick ${ }^{1}$ \\ 1 Department of Physics, University of Washington, \\ Box 351560, Seattle, WA 98195-1560 U. S. A. \\ ${ }^{2}$ Department of Chemistry, Purdue University, \\ West Lafayette, IN 47907-1393 U. S. A.
}

(Dated: July 6, 2021, draft)

\begin{abstract}
We employ a molecular model to study a ternary mixture of saturated lipid, with tails of sixteen carbons, a mono unsaturated lipid with tails of eighteen carbons, and cholesterol. The model, solved within mean-field theory, produces several forms of phase diagrams depending upon the relative strengths of interactions, but only one that shows the coexistence of two liquid phases observed in experiment. The lipids in the phase rich in cholesterol are more ordered than those in the other. The binary cholesterol, saturated lipid system also exhibits liquid, liquid coexistence.
\end{abstract}

PACS numbers: 64.70.Ja,87.16Dg,81.30Dz 
There has been enormous interest in the hypothesis that the lipids comprising the plasma membrane are distributed inhomogeneously, with regions rich in cholesterol and saturated lipids floating, like rafts, in a sea of unsaturated lipids. Such rafts have been implicated in many biological processes including endocytosis, transcription and transduction processes, and viral infection. The extent of the interest is reflected in the number of recent reviews 1, 2, 3, 4, 5]. It has spurred numerous in vitro investigation of ternary systems of cholesterol and a high-melting point (hmp) lipid, either sphingomyelin or a saturated glycerolipid, and a glycerolipid whose melting point is lower, either because it is unsaturated [6, 7, 8, 9] or because, although saturated, it is relatively short [10, 11]. A major question the ternary studies could address is whether the aggregation phenomenon indicated in vivo might simply be attributed to liquid-liquid phase separation. If so, it could be observed in vitro. The assumption was that one fluid, the liquid disordered (ld) phase, would be rich in the lowmelting-point (lmp) lipid, while the other, the liquid ordered (lo) phase, would be rich in cholesterol and the hmp lipid. The nomenclature "liquid ordered" [12] signifies that there are fewer thermally excited gauche bonds in the hydrocarbon tails of the lipid chains in this phase than in the liquid disordered phase. In addition to these two phases, a gel phase occurs below the melting, or main chain transition temperature, of the hmp lipid when the concentration of this lipid is large. This phase is the most ordered of the three.

While there is some agreement on broad features in the interior of the ternary diagrams, there is also considerable uncertainty. In particular the approach to the cholesterol, hmp lipid binary system, and the phase diagram of that system itself remain a matter of controversy. The disagreement centers on whether or not there is coexistence of two liquid phases over some range of temperature even in this binary system.

Evidence of such coexistence derives from several studies using different probes: electron spin resonance [13], freeze fracture electron microscopy [14], nuclear magnetic resonance (NMR) [15], fluorescence resonance energy transfer [16], and other fluorescence studies [9]. Perhaps the strongest evidence, however, is indirect, coming from calorimetry [15, 17]. In the cholesterol, dipalmitoylphosphatidylcholine (DPPC) system, these measurements clearly show a very large and narrow specific heat signal at a temperature which is rather constant over a range of compositions, the standard indicator of a triple point. The signal was so interpreted by Vist and Davis [15]. Given the undisputed existence of a gel phase which coexists with a liquid phase at low temperature, the observation of a triple point leads to 
the conclusion of the existence of a gel and two liquid phases at higher temperatures. There is NMR evidence for such a triple point in another cholesterol, hmp lipid system as well [18].

There are also arguments against the existence of two liquid phases in the cholesterol, hmp lipid system. First, the sharp specific heat signals have been interpreted as not arising from three-phase coexistence by McElhaney and co-workers [17], and they also dispute the conclusion drawn by Vist and Davis from their NMR experiments. Indeed the NMR work of Huang et al. 19] found no such coexistence, nor did those using fluorescence recovery after photo bleaching [20]. Fluorescence microscopy also does not observe phase separation in these systems [7, 8]. Even if the behavior of this binary system were clear, its relation to that of the canonical 1:1:1 raft composition would not be. The question would remain whether the coexistence of liquids in this part of the ternary system was directly related to such coexistence in the binary system.

To clarify the ternary system and its evolution from the binary one, we have carried out a study based upon a microscopic model. Previous theoretical investigations of these mixtures have been limited to the binary systems of cholesterol and hmp lipid [12, 21, 22], or hmp and lmp lipids 23]. Notable among the former is the pioneering study of Ipsen et al. which coined the description "liquid-ordered phase" [12].

Our ternary mixture consists of cholesterol, a lipid with two saturated tails of sixteen carbons, C16:0, and another with two mono-unsaturated tails of eighteen carbons, C18:1. There are essentially two adjustable interaction parameters in the model, and their variation produces three distinct phase diagrams. One is clearly irrelevant to experiment, one is consistent with claims of no liquid, liquid coexistence in the binary system, and one is consistent with claims that there is. Only the last of these exhibits the liquid, liquid coexistence in the heart of the ternary diagram, consistent with experiments.

In our model, the lipids tails are described using the Flory rotational isomeric states representation 24]. One introduces the local volume fraction per unit length for a lipid tail in a particular configuration, $\hat{\phi}_{\sigma}(z)$,

$$
\hat{\phi}_{\sigma}(z)=\sum_{k=1}^{n_{\sigma}} \nu_{\sigma}(k) \delta\left[z-z_{k}\right],
$$

where the $z$ direction is perpendicular to the plane of the bilayer, $\sigma=\mathrm{s}$ or $\mathrm{u}$ is an index indicating whether the lipid is saturated or unsaturated, the $\nu(k)=28 \AA^{3} \equiv \nu_{0}$, the volume of the $k$ 'th monomer if it is a $\mathrm{CH}_{2}$, or $56 \AA^{3}$ if a $\mathrm{CH}_{3}$ group, and $n_{s}=15, n_{u}=17$. The local 
orientation of the chain is conveniently specified by the normal to the plane determined by the $k$ 'th $\mathrm{CH}_{2}$ group,

$$
\mathbf{u}_{\sigma, k}=\frac{\mathbf{r}_{k-1}-\mathbf{r}_{k+1}}{\left|\mathbf{r}_{k-1}-\mathbf{r}_{k+1}\right|}, \quad k=1 \ldots n_{s}-1 .
$$

A similar local volume fraction is introduced for the cholesterol, with index $\sigma=c, n_{c}=27$ and $\nu_{c}(k) \approx 21.0 \AA^{3}$. The orientation of the small acyl chain of the cholesterol is specified in the same manner as are the lipid chains, while the orientation of its rigid rings are specified by the unit vector, $\mathbf{u}_{\mathbf{c}}$, from the third to the seventeenth carbon in the molecule, using the conventional labeling.

In earlier theories, [25, 26], the effect of the short-range repulsive and long-range attractive interactions between elements was accounted for approximately by replacing them by a constraint that the density within the hydrophobic region be constant locally. The free energy of the system can then be written in terms of the $\hat{\phi}$. While accounting well for many properties of bilayers, this theory cannot lead to a main chain transition to a gel phase. Hence we have added 23] an additional separable interaction per unit volume which tends to align the elements with each other and with the bilayer normal c:

$$
\begin{aligned}
V_{\sigma, \sigma^{\prime}} & =-\left(J_{\sigma, \sigma^{\prime}} / \nu_{0}\right) g\left(\mathbf{u}_{\sigma} \cdot \mathbf{c}\right) g\left(\mathbf{u}_{\sigma^{\prime}} \cdot \mathbf{c}\right), \\
g\left(\mathbf{u}_{\sigma} \cdot \mathbf{c}\right) & \equiv(m+1 / 2)\left(\mathbf{u}_{\sigma} \cdot \mathbf{c}\right)^{2 m}
\end{aligned}
$$

For large $m, g \approx m \exp \left(-m \theta^{2}\right)$ where $\theta$ is the angle between the two unit vectors. Matching lipid parameters, we have taken $m=18$. Note that this interaction between two vectors falls off exponentially if either of them is not well aligned with the bilayer normal. We take the strength of the local interactions between bonds in lipid tails to be the same, $J_{s s}=J_{u u}=J_{s u} \equiv J_{l l}$, irrespective of whether the bonds are in a saturated or unsaturated chain, and the interaction strength between the cholesterol and any bond in a lipid tail to be the same, $J_{s c}=J_{u c} \equiv J_{l c}$, irrespective of the type of lipid chain. Thus the model contains three interaction strengths: $J_{l l}$ which is set by the main chain transition temperature, $J_{l c}$, and $J_{c c}$ the strength of the aligning interaction between cholesterols.

Just as the $\hat{\phi}$ describe the local density, so we introduce a function which describes the local ordering with respect to the bilayer normal [27]:

$$
\hat{\xi}_{\sigma}(z)=\sum_{k=1}^{n_{\sigma}-1} \nu_{\sigma}(k) \delta\left(z-z_{k}\right) g\left(\mathbf{u}_{\sigma} \cdot \mathbf{c}\right) .
$$


The Helmholtz free energy per unit area, $f_{A}\left(T, \rho_{s}, \rho_{u}, \rho_{c}\right)$, with $\rho_{\sigma}=N_{\sigma} / A, \sigma=\mathrm{s}$ or $\mathrm{u}$, the areal density of saturated or unsaturated chains, and $\rho_{c}=N_{c} / A$ the areal density of cholesterol, can now be obtained directly within mean-field theory. One finds

$$
\begin{aligned}
& \beta f_{A}=-\frac{1}{\nu_{0}} \int\left\{(\beta / 2) \sum_{\sigma, \sigma^{\prime}} J_{\sigma \sigma^{\prime}} \rho_{\sigma}<\hat{\xi}_{\sigma}>\rho_{\sigma^{\prime}}<\hat{\xi}_{\sigma^{\prime}}>\right. \\
& \left.+\sum_{\sigma} \rho_{\sigma}\left[<\hat{\xi}_{\sigma}>B_{\sigma}(z)+<\hat{\phi}_{\sigma}>\Pi(z)\right]\right\} d z \\
& -\sum_{\sigma} \rho_{\sigma} \ln Q_{\sigma}+\frac{\rho_{s}}{2} \ln \frac{\rho_{s}}{\rho_{0}}+\frac{\rho_{u}}{2} \ln \frac{\rho_{u}}{\rho_{0}}+\rho_{c} \ln \frac{\rho_{c}}{\rho_{0}}
\end{aligned}
$$

with $\rho_{0} \equiv V / A \nu_{0}, \beta \equiv 1 / k_{B} T$. Here $<O_{\sigma}(z)>=\operatorname{Tr} P_{\sigma} \hat{O}_{\sigma}(z)$, the average in a single chain ensemble, with

$$
\begin{aligned}
P_{\sigma} & =\frac{1}{Q_{\sigma}} \exp \left\{-\mathcal{E}_{\sigma}\right\} \equiv \frac{1}{Q_{\sigma}} \exp \left\{-\beta H_{1}\right. \\
& \left.-\frac{1}{\nu_{0}} \int\left[\hat{\phi}_{\sigma}(z) \Pi(z)+\hat{\xi}_{\sigma}(z) B_{\sigma}(z)\right] d z\right\}, \\
Q_{\sigma} & =\operatorname{Tr} \exp \left\{-\mathcal{E}_{\sigma}\right\},
\end{aligned}
$$

and $H_{1}$ contains the intra chain energy arising from the presence of gauche bonds. The three unknown fields $B_{s}=B_{u} \equiv B_{l}, B_{c}$, and $\Pi$ are obtained from minimization of the free energy with respect to the $\left\langle\hat{\xi}_{\sigma}>\right.$ and $<\hat{\phi}_{\sigma}>$ which leads to the three local self consistent equations

$$
\begin{aligned}
1 & =\sum_{\sigma} \rho_{\sigma}<\hat{\phi}_{\sigma}(z)>, \\
B_{l} & =-\beta\left\{J_{l l}\left[\rho_{s}<\hat{\xi}_{s}>+\rho_{u}<\hat{\xi}_{u}>\right]+J_{l c} \rho_{c}<\hat{\xi}_{c}>\right\}, \\
B_{c} & =-\beta\left\{J_{l c}\left[\rho_{s}<\hat{\xi}_{s}>+\rho_{u}<\hat{\xi}_{u}>\right]+J_{c c} \rho_{c}<\hat{\xi}_{c}>\right\} .
\end{aligned}
$$

The first of these is simply the incompressibility constraint.

The heart of the above method, and its difficulty, is the evaluation of the single molecule partition functions $Q_{\sigma}$. To evaluate them, we have generated on the order of $10^{7}$ configurations of each molecule. Finally the effect of the water, lipid interface is taken into account via a contribution to the free energy per unit area of $\gamma_{0}$, set equal to the oil, water tension. Thus the total free energy per unit area, or surface tension, is $f_{\text {tot }}\left(T, \rho_{s}, \rho_{u}, \rho_{c}\right)=f_{A}+\gamma_{0}$. We look for phases for which this surface tension vanishes. Phase equilibria is determined by standard thermodynamic equalities [23].

We now turn to the results of our calculation. The binary phase diagram of unsaturated and saturated lipids [23] depends upon packing constraints and the lipid-lipid interaction 

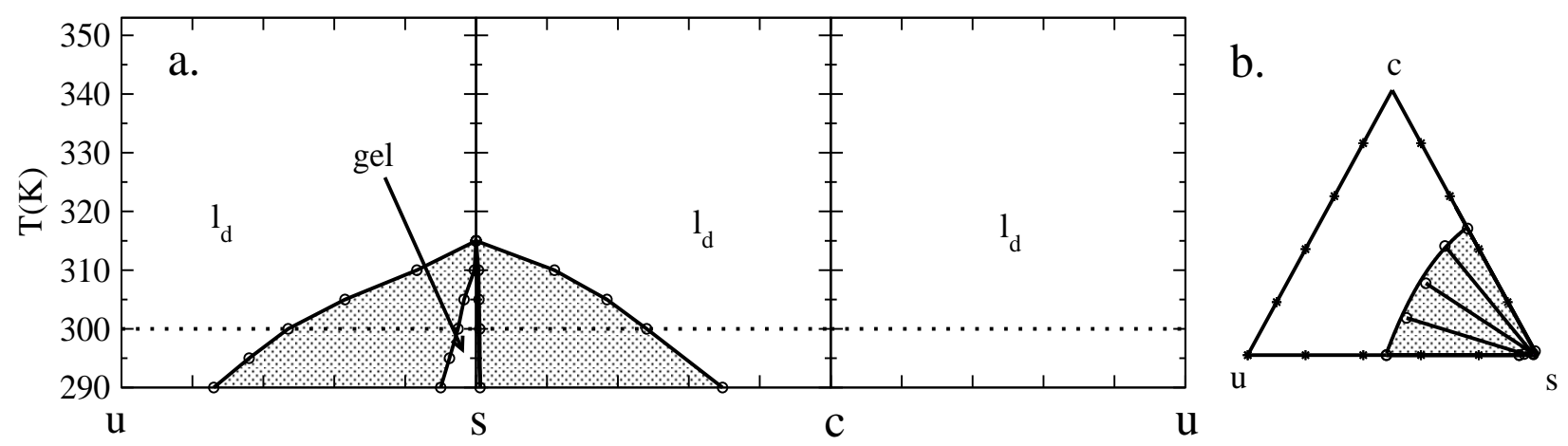

FIG. 1: Calculated phase diagrams of the three binary mixtures of cholesterol (c), saturated (s), and unsaturated $(\mathrm{u})$ lipids in temperature-composition space for $J_{l l}(m+1 / 2)^{2} / k_{B} T^{*}=1.44$ and $J_{l c}=J_{c c}=0.0$. These binary diagrams form the sides of the Gibbs prism, a cut through which at 300K produces the Gibbs triangle shown in Fig. 1b. Regions of two-phase coexistence are shaded, and some tie lines are shown.

strength $J_{l l}$ which we set by matching the main chain transition of the saturated lipid to $T^{*}=315 K$, that of DPPC. Below this temperature there is a gel phase, rich in the saturated lipid, and a disordered liquid phase rich in the unsaturated lipid. The main chain transition temperature of the unsaturated lipid is below $0^{\circ} \mathrm{C}$. The nature of the ternary diagram depends upon the relative strengths, $J_{l c}$ and $J_{c c}$ of the lipid-cholesterol and cholesterolcholesterol aligning interactions. We find essentially three classes of diagrams.

The first occurs when the other two interactions are weak compared to $J_{l l}$. For the binary cholesterol, saturated lipid system, this results only in gel and ld phases, and therefore no liquid, liquid coexistence, consistent with one side of the controversy noted earlier. The three binary phase diagrams as a function of temperature and composition are shown in Fig. 1(a). A ternary diagram at a temperature below the main chain temperature is shown in Fig. 1(b). There is no region of liquid-liquid coexistence. As such regions can be seen directly in the experimental ternary systems [6, 7, 8], this choice of interactions does not apply.

A second class of diagram occurs if the lipid cholesterol aligning interaction, $J_{l c}$, is dominant. This produces a gel phase which is swollen with cholesterol. As there is no experimental evidence for this, we do not consider this choice of interactions further.

A third class of diagram occurs when the interaction between cholesterols is at least comparable to that between lipids. Binary phase diagrams based on our calculations are 

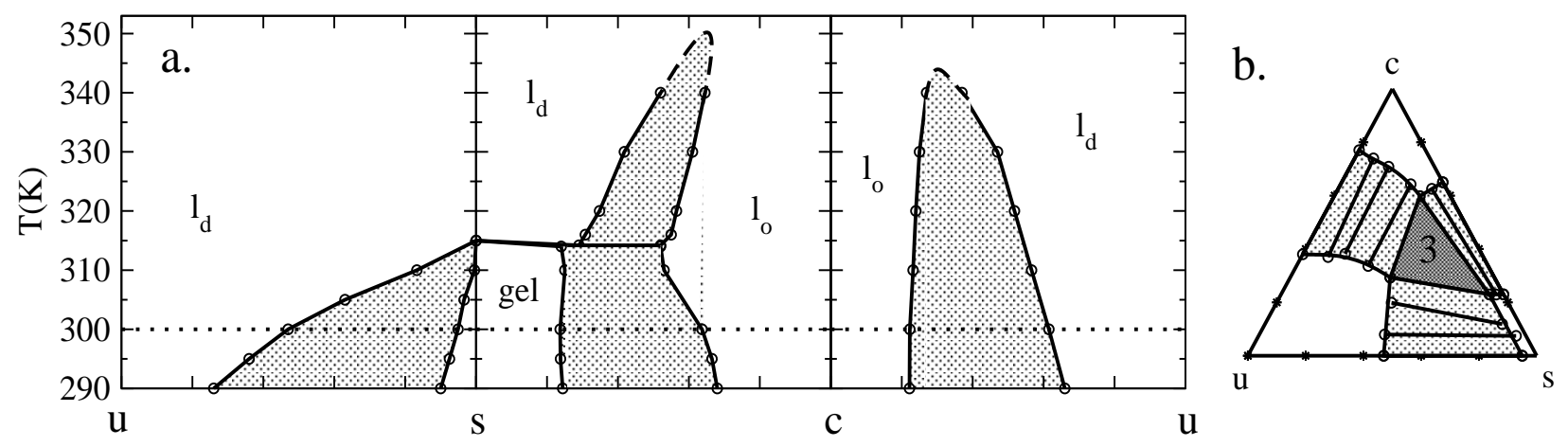

FIG. 2: Figure 2a. Binary phase diagrams for $J_{l c}=0.78 J_{l l}$ and $J_{c c}=0.73 J_{l l}$, and $J_{l l}$ as in Fig. 1. The saturated lipid-cholesterol mixture has a triple point very near the main-chain transition temperature, so that the gel, $l_{d}$ coexistence region is very narrow. Dashed lines are extrapolations. The ternary mixture at $T=300 K$ is shown in Fig. 2 b.

shown in Fig. 2(a). In the cholesterol, saturated lipid system, there is coexistence of two liquid phases over a range of high temperatures, and a temperature of three-phase coexistence with a gel phase, consistent with refs [13]-[16]. A cut through the ternary diagram at a temperature of $T=300 \mathrm{~K}$ below the triple temperature is shown in Fig. 2 (b). There is a large region of coexistence of the lo, ld, and gel phases. There are three regions of two-phase coexistence which extend to the binary axes. The coexistence region between lo and ld liquids would be identified as the region in which rafts could exist. The diagram is topologically identical to that reported in ref [9].

The degree of order, $S_{\mathrm{CD}}$, in the lipid chains is given in terms of the second Legendre polynomial $\left|S_{\mathrm{CD}}\right|=\left|P_{2}\left(\theta_{k}\right)\right| / 2$, where $\theta_{k}$ is the angle between the normals to the bilayer and to the plane of the $k$ 'th $\mathrm{CH}_{2}$ group. The order parameter for the saturated lipids is shown in Fig. 3 for each of the three phases which coexist in the phase diagram of Fig. 2b. As can be seen, the chains in the lo phase are indeed more ordered than those in the ld phase. This increased order is due to the interactions of the lipids with cholesterol, which is itself well ordered. We have also verified that the addition of cholesterol tends to disorder the gel phase, in agreement with experiment [15].

The evolution of the ternary diagram with increasing temperature is simple. The region of three-phase coexistence shrinks and vanishes at the triple temperature of the binary cholesterol, hmp lipid system. Above the main chain temperature of this lipid, there is a two- 


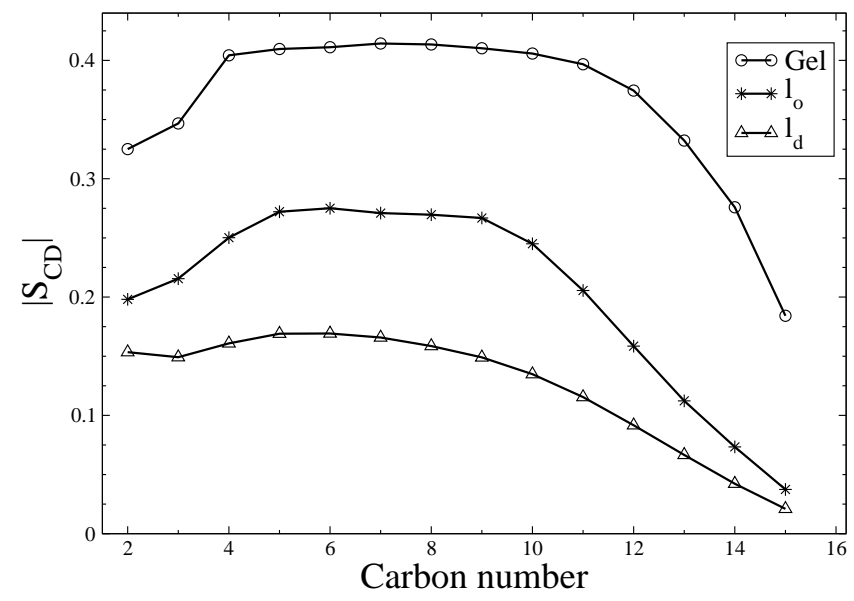

FIG. 3: The order parameters for the saturated tails in the three coexisting phases of the ternary mixture at $300 \mathrm{~K}$. Cholesterol concentration is greatest in the lo phase, which is more ordered than the ld phase. The effect of the larger cholesterol concentration is evident in the plateau corresponding to ordering about cholesterol's ring structure.

phase region of lo and ld coexistence which stretches from one binary system with cholesterol to the other. At higher temperatures, this region detaches from the binary cholesterol, lmp lipid axis at a critical point, and the region shrinks with further increase of temperature until it vanishes at a critical point on the cholesterol, hmp lipid axis. It is clear from the evolution of our calculated ternary diagram that the liquid, liquid coexistence within our ternary diagram is intimately tied to a similar coexistence in the binary cholesterol, saturated lipid system. Given that the coexistence of liquids has been observed in the ternary systems, the calculation gives strong support to the view that such coexistence already exists in the cholesterol, hmp lipid system as well.

We are grateful to Sarah Keller and Sarah Veatch for numerous profitable discussions. This work was supported by the National Science Foundation under Grants No. DMR0140500 and CTS-0338377.

[1] K. Simons and D. Toomre, Nature Revs. Mol. Cell. Biol. 1, 31 (2000).

[2] M. Edidin, Annu. Rev. Biophys. Biomol. Struct. 32, 257 (2003).

[3] S. Munro, Cell 115, 377 (2003). 
[4] K. Simons and W. Vaz, Annu. Rev. Biophys. Biomol. Struct. 33, 269 (2004).

[5] T. McMullen, R. N. Lewis, and R. McElhaney, Curr. Op. Coll. Int. Sci. 8, 459 (2004).

[6] C. Dietrich, et al., Biophys. J. 80, 1417 (2001).

[7] S. L. Veatch and S. L. Keller, Phys. Rev. Lett. 89, 268101 (2002).

[8] S. L. Veatch and S. L. Keller, Biophys. J. 85, 3074 (2003).

[9] R. de Almeida, A. Fedorov, and M. Prieto, Biophys. J. 85, 2406 (2003).

[10] J. Silvius, D. del Giudice, and M. Lafleur, Biochemistry 35, 15198 (1996).

[11] G. Feigenson and J. Buboltz, Biophys. J. 80, 2775 (2001).

[12] J. H. Ipsen, et al., Biochim. Biophys. Acta. 905, 162 (1987).

[13] E. Shimshick and H. M. McConnell, Biochim. Biophys. Res. Comm. 53, 446 (1973).

[14] B. R. Lentz, D. A. Barrow, and M. Hoechli, Biochemistry 19, 1943 (1980).

[15] M. Vist and J. Davis, Biochemistry 29, 451 (1990).

[16] L. Loura, A. Fedorov, and M. Prieto, Biophys. J. 80, 776 (2001).

[17] T. McMullen and R. McElhaney, Biochim. Biphys. Acta 1234, 90 (1995).

[18] L. Miao, et al.,Biophys. J. 82, 1429 (2002).

[19] T.-H. Huang, et al., Biochemistry 32, 13277 (1993).

[20] J. Rubenstein, B. Smith, and H. McConnell, Proc. Natl. Acad. Sci. U S A 76, 15 (1979).

[21] M. Nielsen, et al., Phys. Rev. E. 59, 5790 (1999).

[22] S. Komura, et al., Europhys. Lett. 67, 321 (2004).

[23] R. Elliott, et al., J. Chem. Phys. 122, 0449041 (2005).

[24] P. J. Flory, Statistical Mechanics of Chain Molecules (Wiley-Interscience, New York, 1969).

[25] D. W. R. Gruen, Biochim. Biophys. Acta 595, 161 (1979).

[26] I. Szleifer, A. Ben-Shaul, and W. M. Gelbart, J. Chem. Phys. 85, 5345 (1986).

[27] F. Schmid, J. Phys.: Condens. Matter 10, 8105 (1998). 